\title{
Inner product spaces and quadratic functional equations
}

\author{
Jae-Hyeong Bae ${ }^{1}$, Batool Noori², M.B. Moghimi and Abbas Najati ${ }^{2 *}$ (D)
}

\author{
"Correspondence: \\ a.nejati@yahoo.com \\ ${ }^{2}$ Department of Mathematics, \\ Faculty of Sciences, University of \\ Mohaghegh Ardabili, Ardabil, Iran \\ Full list of author information is \\ available at the end of the article
}

$$
\begin{aligned}
& \text { Abstract } \\
& \text { In this paper, we introduce the functional equations } \\
& \qquad \begin{aligned}
f(2 x-y)+f(x+2 y) & =5[f(x)+f(y)] \\
f(2 x-y)+f(x+2 y) & =5 f(x)+4 f(y)+f(-y) \\
f(2 x-y)+f(x+2 y) & =5 f(x)+f(2 y)+f(-y) \\
f(2 x-y)+f(x+2 y) & =4[f(x)+f(y)]+[f(-x)+f(-y)]
\end{aligned}
\end{aligned}
$$

We show that these functional equations are quadratic and apply them to characterization of inner product spaces. We also investigate the stability problem on restricted domains. These results are applied to study the asymptotic behaviors of these quadratic functions in complete $\beta$-normed spaces.

MSC: 39B82; 39B52; 39B62

Keywords: Stability; Quadratic functional equation; Quadratic function; Asymptotic behavior

\section{Introduction}

A function $f: V \rightarrow W$ between linear vector spaces $V$ and $W$ is called a quadratic function if

$$
f(x+y)+f(x-y)=2 f(x)+2 f(y), \quad x, y \in V .
$$

It is clear that $f: \mathbb{R} \rightarrow \mathbb{R}$ defined by $f(x)=a x^{2}$ is a quadratic function, where $a$ is an arbitrary constant. Indeed, a continuous quadratic function $f: \mathbb{R} \rightarrow \mathbb{R}$ is of this form. It is well known that a function $f: V \rightarrow W$ between real vector spaces $V$ and $W$ is quadratic if and only if there exists a unique symmetric biadditive function $B: V \times V \rightarrow W$ such that $f(x)=B(x, x)$ for all $x \in V$ (see [1-3]). The functional equation (1.1) plays an important role in the characterization of inner product spaces [4]. In this paper, we deal with the functional equation

$$
f(2 x-y)+f(x+2 y)=5[f(x)+f(y)]
$$

(c) The Author(s) 2021. This article is licensed under a Creative Commons Attribution 4.0 International License, which permits use sharing, adaptation, distribution and reproduction in any medium or format, as long as you give appropriate credit to the original author(s) and the source, provide a link to the Creative Commons licence, and indicate if changes were made. The images or other third party material in this article are included in the article's Creative Commons licence, unless indicated otherwise in a credit line to the material. If material is not included in the article's Creative Commons licence and your intended use is not permitted by statutory regulation or exceeds the permitted use, you will need to obtain permission directly from the copyright holder. To view a copy of this licence, visit http://creativecommons.org/licenses/by/4.0/. 
We also consider some functional equations with slight changes in the functional equation (1.2):

$$
\begin{aligned}
& f(2 x-y)+f(x+2 y)=5 f(x)+4 f(y)+f(-y), \\
& f(2 x-y)+f(x+2 y)=5 f(x)+f(2 y)+f(-y), \\
& f(2 x-y)+f(x+2 y)=4[f(x)+f(y)]+[f(-x)+f(-y)] .
\end{aligned}
$$

The stability of the quadratic functional equation (1.1) was first investigated by Skof [5]. Czerwik [6] studied the stability of the quadratic functional equation (1.1) in a general case and generalized Skof's result. For more detailed information on the stability results of the functional equation (1.1) and other quadratic functional equations, we refer the readers to [7-17]. We also refer the readers to the books $[1,2,18-20]$.

\section{Solutions of Eq. (1.2)}

In this section, we show that the functional equation (1.2) is equivalent to the quadratic equation (1.1). That is, every solution of Eq. (1.2) is a quadratic function.

Theorem 2.1 Let $X$ and $Y$ be real vector spaces. A function $f: X \rightarrow Y$ satisfies (1.2) if and only iff satisfies (1.1).

Proof Let $f$ satisfy (1.2). Replacing $x=y=0$ in (1.2), we obtain $f(0)=0$. Letting $y=0$ in (1.2), we get $f(2 x)=4 f(x)$ for all $x \in X$. Then if we put $x=0$ in (1.2), we infer that $f$ is even. Replacing $x$ in (1.2) by $x-y$ yields

$$
f(2 x-3 y)+f(x+y)=5[f(x-y)+f(y)], \quad x, y \in X .
$$

Replacing $y$ in (1.2) by $2 y-x$ yields

$$
f(3 x-2 y)+f(4 y-x)=5[f(x)+f(2 y-x)], \quad x, y \in X .
$$

Interchanging the role of $x$ and $y$, we obtain

$$
f(3 y-2 x)+f(4 x-y)=5[f(y)+f(2 x-y)], \quad x, y \in X .
$$

Subtracting (2.1) from (2.2) and using the evenness of $f$, we get

$$
f(4 x-y)-f(x+y)=5[f(2 x-y)-f(x-y)], \quad x, y \in X .
$$

Replacing $x$ by $2 x$ in (1.2) and using $f(2 x)=4 f(x)$, we obtain

$$
f(4 x-y)+4 f(x+y)=5[4 f(x)+f(y)], \quad x, y \in X .
$$

Subtracting (2.4) from (2.2), we get

$$
f(3 y-2 x)-4 f(x+y)=5[f(2 x-y)-4 f(x)], \quad x, y \in X .
$$


Subtracting (2.5) from (2.1) and using the evenness of $f$, we get

$$
f(x+y)=f(x-y)+f(y)-f(2 x-y)+4 f(x), \quad x, y \in X .
$$

Replacing $y$ by $2 y$ in (2.6) and using $f(2 x)=4 f(x)$, we obtain

$$
f(x+2 y)=f(x-2 y)+4 f(y)-4 f(x-y)+4 f(x), \quad x, y \in X .
$$

If we replace $y$ in (2.7) with $-y$ and add the resultant equation to (2.7), then we obtain

$$
f(x+y)+f(x-y)=2 f(x)+2 f(y), \quad x, y \in X .
$$

Hence $f$ is quadratic. Conversely, if $f$ is quadratic, then there exists a symmetric biadditive function $B: X^{2} \rightarrow X$ such that $f(x)=B(x, x)$ for all $x \in X$ [21, Theorem 9.5]. Hence it is easy to see that $f$ satisfies (1.2).

Corollary 2.2 Let $f: X \rightarrow Y$. The following are equivalent:

(i) $f(2 x-y)+f(x+2 y)=5 f(x)+4 f(y)+f(-y), x, y \in X$;

(ii) $f(2 x-y)+f(x+2 y)=4[f(x)+f(y)]+[f(-x)+f(-y)], x, y \in X$;

(iii) $f(2 x-y)+f(x+2 y)=5 f(x)+f(2 y)+f(-y), x, y \in X$;

(iv) $f(2 x-y)+f(x+2 y)=5[f(x)+f(y)], x, y \in X$;

(v) $f$ is quadratic.

Proof (i) $\Rightarrow$ (iv). Letting $x=y=0$ in (i), we get $f(0)=0$. Putting $y=0$ into (i), we obtain $f(2 x)=4 f(x)$ for all $x \in X$. First put $y=x$ into (i) and then $y=-x$ into (i) to get $f(3 x)=$ $8 f(x)+f(-x)$ and $f(3 x)=6 f(x)+3 f(-x)$. So, we infer that $f$ is even. Consequently, $f$ satisfies (iv). To prove (ii) $\Rightarrow$ (iv), letting $x=0$ in (ii) and using $f(0)=0$, we get $f(2 y)=4 f(y)$. Letting $y=0$ in (ii), we get that $f$ is even, and consequently $f$ satisfies (iv). To prove (iii) $\Rightarrow$ (i), letting $y=0$ in (iii) and using $f(0)=0$, we get $f(2 x)=4 f(x)$ for all $x \in X$. Then $f$ satisfies (i). The equivalence of conditions (iv) and (v) follows from Theorem 2.1. The implications (iv) $\Rightarrow$ (i), (ii), (iii) are straightforward because (iv) implies that $f$ is even and $f(2 x)=4 f(x)$ for all $x \in X$. Hence the proof is completed.

Proposition 2.3 Let $\mathcal{X}$ be a normed linear space with norm $\|\cdot\|$. Then $\mathcal{X}$ is an inner product space with respect to $\|\cdot\|$ if and only if

$$
\|2 x-y\|^{2}+\|x+2 y\|^{2}=5\left(\|x\|^{2}+\|y\|^{2}\right), \quad x, y \in \mathcal{X} .
$$

Proof Let $f: \mathcal{X} \rightarrow \mathbb{R}$ be a function defined by $f(x)=\|x\|^{2}$. If $\mathcal{X}$ is an inner product space, then clearly (2.8) holds for all $x, y \in \mathcal{X}$. Conversely, if (2.8) holds, then the function $f$ satisfies (1.2). By Theorem 2.1 the function $f$ satisfies (1.1). This means that

$$
\|x+y\|^{2}+\|x-y\|^{2}=2\|x\|^{2}+2\|y\|^{2}, \quad x, y \in \mathcal{X} .
$$

Therefore $\mathcal{X}$ is an inner product space (see [4]). 
Proposition 2.4 Let $\mathcal{Y}$ be a real vector space, and let $\varphi: \mathcal{Y} \rightarrow[0,+\infty)$ be a function satisfying

(i) $\varphi(x)=0$ iff $x=0$;

(ii) $\sqrt{\varphi(t x)}=|t| \sqrt{\varphi(x)}, t \in \mathbb{R}, x \in \mathcal{Y}$;

(iii) $\varphi(2 x-y)+\varphi(x+2 y)=5[\varphi(x)+\varphi(y)], x, y \in \mathcal{Y}$.

Then $\mathcal{Y}$ is an inner product space.

Proof The proof of Theorem 2.1 shows that $\varphi$ satisfies (1.1). Then $\mathcal{Y}$ is an inner product space by [22, Theorem 3.1].

Proposition 2.5 Let $p, q, r, s \in(0,+\infty)$, and let $\mathcal{X}$ be a normed linear space with norm $\|\cdot\|$. Suppose that

$$
\|2 x-y\|^{p}+\|x+2 y\|^{q}=5\left(\|x\|^{r}+\|y\|^{s}\right), \quad x, y \in \mathcal{X} .
$$

Then $p=q=r=s=2$.

Proof Setting $y=0$ in (2.9), we get

$$
2^{p}\|x\|^{p}+\|x\|^{q}=5\|x\|^{r}, \quad x \in \mathcal{X} .
$$

If we take $x \in \mathcal{X}$ with $\|x\|=1$ in (2.10), then we get $p=2$. Letting $y=x$ in (2.9), we get

$$
\|x\|^{p}+3^{q}\|x\|^{q}=5\left(\|x\|^{r}+\|x\|^{s}\right), \quad x \in \mathcal{X} .
$$

Setting $\|x\|=1$ in (2.11), we get $q=2$. If we put $y=2 x$ with $\|x\|=1$ in (2.9) and use $p=q=2$, then we obtain $s=2$. Finally, letting $x=2 y$ with $\|y\|=1$ in (2.9) and using $p=q=s=2$, we obtain $r=2$. This completes the proof.

Corollary 2.6 Let $\mathcal{X}$ be a normed linear space with norm $\|\cdot\|$. Then $\mathcal{X}$ is an inner product space if and only if there exist $p, q, r, s \in(0,+\infty)$ such that

$$
\|2 x-y\|^{p}+\|x+2 y\|^{q}=5\left(\|x\|^{r}+\|y\|^{s}\right), \quad x, y \in \mathcal{X} .
$$

\section{Stability of Eq. (1.2): fixed point method}

Let $\mathcal{X}$ be a nonempty set. A function $d: \mathcal{X} \times \mathcal{X} \rightarrow[0,+\infty]$ is called a generalized metric on $\mathcal{X}$ if it satisfies

(1) $d(x, y)=0$ if and only if $x=y$;

(2) $d(x, y)=d(y, x)$ for all $x, y \in \mathcal{X}$;

(3) $d(x, z) \leq d(x, y)+d(y, z)$ for all $x, y, z \in \mathcal{X}$.

It is clear that the only difference between the generalized metric and the metric is that the generalized metric accepts the infinity. We will use the following fundamental theorem in fixed point theory.

Theorem 3.1 ([23]) Let $(\mathcal{X}, d)$ be a generalized complete metric space, and let $\Lambda: \mathcal{X} \rightarrow \mathcal{X}$ be a strictly contractive function with Lipschitz constant $0<L<1$. Suppose that for an element $a \in \mathcal{X}$, there exists a nonnegative integer $k$ such that $d\left(\Lambda^{k+1} a, \Lambda^{k} a\right)<\infty$. Then 
(i) the sequence $\left\{\Lambda^{n} a\right\}_{n=1}^{\infty}$ converges to a fixed point $b \in \mathcal{X}$ of $\Lambda$;

(ii) $b$ is the unique fixed point of $\Lambda$ in the set $\mathcal{Y}=\left\{y \in \mathcal{X}: d\left(\Lambda^{k} a, y\right)<\infty\right\}$;

(iii) $d(y, b) \leq \frac{1}{1-L} d(y, \Lambda y)$ for all $y \in \mathcal{Y}$.

In this section, $0<\beta \leq 1$ is a fixed real number. Suppose $E$ is a real vector space. A function $\|\cdot\|_{\beta}: E \rightarrow[0,+\infty)$ is called a $\beta$-norm if it satisfies

(B1) $\|x\|_{\beta}=0$ if and only if $x=0$;

(B2) $\|\lambda x\|_{\beta}=|\lambda|^{\beta}\|x\|_{\beta}$ for all $\lambda \in \mathbb{R}$ and $x \in E$;

(B3) $\|x+y\|_{\beta} \leq\|x\|_{\beta}+\|y\|_{\beta}$ for all $x, y \in E$.

$\left(E,\|\cdot\|_{\beta}\right)$ is called a complete $\beta$-normed space if every Cauchy sequence $\left\{x_{n}\right\}_{n=1}^{\infty} \subseteq E$ (with respect to $\|\cdot\|_{\beta}$ ) converges to an element $x \in E$. Using the idea of Cădariu and Radu [24], we apply the fixed point method to study the stability of quadratic functional equation (1.2).

Theorem 3.2 Let $X$ be a linear space, and let $E$ be a complete $\beta$-normed space. Suppose $\varphi: X^{2} \rightarrow[0,+\infty)$ is a given function and there exists a constant $0<L<1$ such that

$$
\lim _{n \rightarrow \infty} \frac{\varphi\left(3^{n} x, 3^{n} y\right)}{9^{n \beta}}=0, \quad \varphi(3 x, 3 x) \leq 9^{\beta} L \varphi(x, x), \quad x, y \in X .
$$

Furthermore, let $f: X \rightarrow E$ be a function that satisfies

$$
\|f(2 x-y)+f(x+2 y)-5 f(x)-5 f(y)\|_{\beta} \leq \varphi(x, y), \quad x, y \in X .
$$

Then there exists a unique quadratic function $Q: X \rightarrow E$ such that

$$
\|f(x)-Q(x)\|_{\beta} \leq \frac{1}{9^{\beta}(1-L)} \varphi(x, x), \quad x \in X
$$

Proof Let $\Omega=\{g: X \rightarrow E\}$. Define $d: \Omega^{2} \rightarrow[0,+\infty]$ by

$$
d(g, h)=\inf \left\{C \in[0,+\infty]:\|g(x)-h(x)\|_{\beta} \leq C \varphi(x, x), x \in X\right\} .
$$

We easily to see that $d$ is a generalized metric on $\Omega$ and $(\Omega, d)$ is complete. We define the function $T: \Omega \rightarrow \Omega$ by $(T g)(x)=\frac{1}{9} g(3 x)$ for all $x \in X$. We show that $d(T g, T h) \leq L d(g, h)$ for all $g, h \in \Omega$. Let $g, h \in \Omega$ be such that $d(g, h)<\infty$, and take arbitrary $\varepsilon>0$. Then $\| g(x)-$ $h(x) \|_{\beta} \leq C \varphi(x, x)$ for all $x \in X$, where $C=d(g, h)+\varepsilon$. Replacing $x$ by $3 x$ in the last inequality and applying (3.1), we get

$$
\|g(3 x)-h(3 x)\|_{\beta} \leq C 9^{\beta} L \varphi(x, x), \quad x \in X
$$

This means that $d(T g, T h) \leq L C=L(d(g, h)+\varepsilon)$, and since $\varepsilon>0$ is arbitrary, we infer that $d(T g, T h) \leq L d(g, h)$. So $T$ is strictly contractive on $\Omega$ with the Lipschitz constant $L$. On the other hand, replacing $y=x$ in (3.2) and dividing both sides by $9^{\beta}$, we obtain

$$
\|T f(x)-f(x)\|_{\beta} \leq \frac{\varphi(x, x)}{9^{\beta}}, \quad x \in X .
$$


Hence $d(T f, f) \leq 9^{-\beta}<\infty$. By (i) of Theorem 3.1 we conclude that the sequence $\left\{T^{n} f\right\}_{n=1}^{\infty}$ converges to a fixed point $Q \in \Omega$ of $T$. Hence

$$
Q: X \rightarrow E, \quad Q(x)=\lim _{n \rightarrow \infty} \frac{f\left(3^{n} x\right)}{9^{n}}, \quad x \in X
$$

Moreover, by (ii) and (iii) of Theorem 3.1 $Q$ is the unique fixed point of $\Delta:=\{g \in \Omega$ : $d(g, f)<\infty\}$, and

$$
d(f, Q) \leq \frac{1}{1-L} d(T f, f) \leq \frac{1}{9^{\beta}(1-L)}
$$

Therefore inequality (3.3) holds. Using (3.1), (3.2), and (3.4), we get

$$
\begin{aligned}
& \|Q(2 x-y)+Q(x+2 y)-5 Q(x)-5 Q(y)\|_{\beta} \\
& \quad=\lim _{n \rightarrow \infty}\left\|\frac{f\left(3^{n}(2 x-y)\right)}{9^{n}}+\frac{f\left(3^{n}(x+2 y)\right)}{9^{n}}-5 \frac{f\left(3^{n} x\right)}{9^{n}}-5 \frac{f\left(3^{n} y\right)}{9^{n}}\right\|_{\beta} \\
& \quad \leq \lim _{n \rightarrow \infty} \frac{\varphi\left(3^{n} x, 3^{n} y\right)}{9^{n \beta}}=0, \quad x, y \in X .
\end{aligned}
$$

So $Q$ satisfies (1.2), and, consequently, $Q$ is quadratic by Theorem 2.1. The uniqueness of $Q$ is a well-known and simple consequence of (3.3).

Similarly, we have the following result.

Theorem 3.3 Let $X$ be a linear space, and let $E$ be a complete $\beta$-normed space. Suppose $\varphi: X^{2} \rightarrow[0,+\infty)$ is a given function and there exists a constant $0<L<1$ such that

$$
\lim _{n \rightarrow \infty} 9^{n \beta} \varphi\left(\frac{x}{3^{n}}, \frac{y}{3^{n}}\right)=0, \quad 9^{\beta} \varphi(x, x) \leq L \varphi(3 x, 3 x), \quad x, y \in X .
$$

Furthermore, let $f: X \rightarrow E$ be a function that satisfies

$$
\|f(2 x-y)+f(x+2 y)-5 f(x)-5 f(y)\|_{\beta} \leq \varphi(x, y), \quad x, y \in X .
$$

Then there exists a unique quadratic function $Q: X \rightarrow E$ such that

$$
\|f(x)-Q(x)\|_{\beta} \leq \frac{L}{9^{\beta}(1-L)} \varphi(x, x), \quad x \in X .
$$

Corollary 3.4 Let $X$ be a normed space, and let $E$ be a complete $\beta$-normed space. Suppose $\delta, \varepsilon \geq 0, p, q \in \mathbb{R}$, and $f: X \rightarrow E$ is a given function such that

$$
\|f(2 x-y)+f(x+2 y)-5 f(x)-5 f(y)\|_{\beta} \leq \begin{cases}\delta+\varepsilon\left(\|x\|^{p}+\|y\|^{q}\right), & p, q<2 \beta \\ \varepsilon\left(\|x\|^{p}+\|y\|^{q}\right), & p, q>2 \beta\end{cases}
$$


for all $x, y \in X$ (resp., for all $x, y \in X \backslash\{0\}$ when at least $p<0$ or $q<0)$. Then there exists a unique quadratic function $Q: X \rightarrow E$ such that

$$
\|f(x)-Q(x)\|_{\beta} \leq \begin{cases}\frac{\delta+\varepsilon\left(\|x\|^{p}+\|x\|^{q}\right)}{9^{\beta}-3^{r}}, & p, q<2 \beta, \\ \frac{3^{s} \varepsilon}{3^{s}-9^{\beta}}\left(\|x\|^{p}+\|x\|^{q}\right), & p, q>2 \beta,\end{cases}
$$

for all $x \in X$ (resp., for all $x \in X \backslash\{0\}$ when at least $p<0$ or $q<0$ ), where $r=\max \{|p|,|q|\}$ and $s=\min \{p, q\}$.

Theorem 3.5 Let $X$ be a linear space, and let $E$ be a complete $\beta$-normed space. Suppose functions $f: X \rightarrow E$ and $\varphi: X^{2} \rightarrow[0,+\infty)$ are such that

$$
\|f(2 x-y)+f(x+2 y)-5 f(x)-5 f(y)\|_{\beta} \leq \varphi(x, y), \quad x, y \in X .
$$

Then $f$ is quadratic if we have one of the following possibilities:

(i) $\lim _{n \rightarrow \infty} \frac{\varphi\left(2^{n} x, 2^{n} y\right)}{4^{n \beta}}=0, \varphi(x, 0)=0, x, y \in X$;

(ii) $\lim _{n \rightarrow \infty} 4^{n \beta} \varphi\left(\frac{x}{2^{n}}, \frac{y}{2^{n}}\right)=0, \varphi(x, 0)=0, x, y \in X$.

Proof Letting $y=0$ in (3.5), we get $f(2 x)-4 f(x)=5 f(0)$ for all $x \in X$. Then $f(0)=0$, and

$$
\frac{f\left(2^{n} x\right)}{4^{n}}=f(x), \quad 4^{n} f\left(\frac{x}{2^{n}}\right)=f(x), \quad x \in X, n \in \mathbb{N} .
$$

In case (i), replacing $x, y$ by $2^{n} x, 2^{n} y$ in (3.5), respectively, and using (3.6), we obtain

$$
\|f(2 x-y)+f(x+2 y)-5 f(x)-5 f(y)\|_{\beta} \leq \frac{\varphi\left(2^{n} x, 2^{n} y\right)}{4^{n \beta}}, \quad x, y \in X
$$

Taking the limit in (3.7) as $n \rightarrow \infty$ yields $f(2 x-y)+f(x+2 y)=5 f(x)+5 f(y)$ for all $x, y \in X$. Consequently, $f$ is quadratic by Theorem 2.1.

Similarly, we get the result in case (ii).

Corollary 3.6 Let $X$ be a normed space, and let $E$ be a complete $\beta$-normed space. Suppose $p, q>0$ with $p+q \neq 2 \beta$ and $f: X \rightarrow E$ is a function such that

$$
\|f(2 x-y)+f(x+2 y)-5 f(x)-5 f(y)\|_{\beta} \leq\|x\|^{p}\|y\|^{q}, \quad x, y \in X .
$$

Then $f$ is quadratic.

\section{Stability of Eq. (1.2) on restricted domains}

In this section, we investigate the stability of the functional equation (1.2) on a restricted domain. As an application, we use the result to the study of an interesting asymptotic behavior of that equation. It should be mentioned that Skof [5] was the first author to treat the stability of the quadratic equation. Czerwik [6] proved a stability theorem on the quadratic equation. As a particular case, he proved the following theorem. 
Theorem 4.1 Let $X$ be a vector space, let $Y$ be a Banach space, and let $\delta \geq 0$. If afunction $f: X \rightarrow Y$ satisfies the inequality

$$
\|f(x+y)+f(x-y)-2 f(x)-2 f(y)\| \leq \delta
$$

for all $x, y \in X$, then there exists a unique quadratic function $Q: X \rightarrow Y$ such that $\| f(x)-$ $Q(x) \| \leq \frac{\delta}{2}$ for all $x \in X$. Moreover, iff is measurable or if $f(t x)$ is continuous in $t$ for each fixed $x \in X$, then $Q(t x)=t^{2} Q(x)$ for all $x \in X$ and $t \in \mathbb{R}$.

In the following results, we assume that $X$ is a normed space and $Y$ is a complete $\beta$ normed space.

Theorem 4.2 Let $d>0$ and $\delta \geq 0$. Assume that a function $f: X \rightarrow Y$ satisfies the inequality

$$
\|f(2 x-y)+f(x+2 y)-5 f(x)-5 f(y)\|_{\beta} \leq \delta
$$

for all $x, y \in X$ with $\|x\|+\|y\| \geq d$. Then there exists a unique quadratic function $Q: X \rightarrow Y$ such that

$$
\|f(x)-Q(x)\|_{\beta} \leq \frac{9^{\beta}+5^{\beta}+1}{5^{\beta}\left(9^{\beta}-1\right)} \delta, \quad x \in X .
$$

Proof Letting $y=x$ in (4.1), we get

$$
\|f(3 x)-9 f(x)\|_{\beta} \leq \delta, \quad\|x\| \geq d .
$$

Replacing $x$ by $3^{n} x$ in this inequality and dividing by $9^{\beta(n+1)}$, we get

$$
\left\|\frac{f\left(3^{n+1} x\right)}{9^{n+1}}-\frac{f\left(3^{n} x\right)}{9^{n}}\right\|_{\beta} \leq \frac{\delta}{9^{\beta(n+1)}}, \quad\|x\| \geq d .
$$

Then

$$
\left\|\frac{f\left(3^{n+1} x\right)}{9^{n+1}}-\frac{f\left(3^{m} x\right)}{9^{m}}\right\|_{\beta} \leq \sum_{k=m}^{n} \frac{\delta}{9^{\beta(k+1)}}, \quad\|x\| \geq d, n \geq m \geq 0 .
$$

This shows that $\left\{\frac{f\left(3^{n} x\right)}{9^{n}}\right\}_{n=1}^{\infty}$ is a Cauchy sequence for each $x \in X$. As $Y$ is complete, we can define the function $Q: X \rightarrow Y$ by

$$
Q(x):=\lim _{n \rightarrow \infty} \frac{f\left(3^{n} x\right)}{9^{n}}, \quad x \in X .
$$

Hence (4.1) implies that

$$
Q(2 x-y)+Q(x+2 y)=5 Q(x)+5 Q(y), \quad x, y \in X .
$$

Consequently, $Q$ is quadratic by Theorem 2.1. Letting $m=0$ and taking the limit as $n \rightarrow \infty$ in (4.3), we obtain

$$
\|Q(x)-f(x)\|_{\beta} \leq \frac{\delta}{9^{\beta}-1}, \quad\|x\| \geq d .
$$


We now let $x, y \in X$ with $x \neq 0$ and choose $m \in \mathbb{N}$ such that $\left\|3^{m} x\right\| \geq d+2\|y\|$. Then (4.1) and (4.5) imply

$$
\begin{aligned}
& \left\|f\left(2 \times 3^{m} x-y\right)+f\left(3^{m} x+2 y\right)-5 f\left(3^{m} x\right)-5 f(y)\right\|_{\beta} \leq \delta, \\
& \left\|Q\left(2 \times 3^{m} x-y\right)-f\left(2 \times 3^{m} x-y\right)\right\|_{\beta} \leq \frac{\delta}{9^{\beta}-1}, \\
& \left\|Q\left(3^{m} x+2 y\right)-f\left(3^{m} x+2 y\right)\right\|_{\beta} \leq \frac{\delta}{9^{\beta}-1}, \\
& \left\|5 f\left(3^{m} x\right)-5 Q\left(3^{m} x\right)\right\|_{\beta} \leq \frac{5^{\beta} \delta}{9^{\beta}-1} .
\end{aligned}
$$

Since $Q$ satisfies (4.4), using the triangle inequality for these inequalities, we get

$$
\|f(y)-Q(y)\|_{\beta} \leq \frac{9^{\beta}+5^{\beta}+1}{5^{\beta}\left(9^{\beta}-1\right)} \delta .
$$

It remains to prove the uniqueness of $Q$. Assume that $S: X \rightarrow X$ is another quadratic function that satisfies inequality (4.2). Then we have

$$
\|Q(x)-S(x)\|_{\beta} \leq\|Q(x)-f(x)\|_{\beta}+\|f(x)-S(x)\|_{\beta} \leq \frac{2\left(9^{\beta}+5^{\beta}+1\right)}{5^{\beta}\left(9^{\beta}-1\right)} \delta, \quad x \in X .
$$

Since $Q$ and $S$ are quadratic, the last inequality implies that

$$
\|Q(x)-S(x)\|_{\beta}=\frac{1}{n^{2 \beta}}\|Q(n x)-S(n x)\|_{\beta} \leq \frac{2\left(9^{\beta}+5^{\beta}+1\right)}{\left(5 n^{2}\right)^{\beta}\left(9^{\beta}-1\right)} \delta, \quad x \in X, n \in \mathbb{N} .
$$

Taking the limit as $n \rightarrow \infty$, we obtain $Q(x)=S(x)$ for all $x \in X$. This ends the proof.

Corollary 4.3 Let $d>0$ and $\delta \geq 0$. Assume that a function $f: X \rightarrow Y$ satisfies the inequality

$$
\|f(2 x-y)+f(x+2 y)-5 f(x)-5 f(y)\|_{\beta} \leq \delta, \quad \max \{\|x\|,\|y\|\} \geq d .
$$

Then there exists a unique quadratic function $Q: X \rightarrow Y$ satisfying (4.2).

Proof If $\|x\|+\|y\| \geq 2 d$, then $\max \{\|x\|,\|y\|\} \geq d$. Hence $f$ satisfies (4.1) for all $x, y \in X$ with $\|x\|+\|y\| \geq 2 d$. Therefore the result follows from Theorem 4.2.

We apply these results to study the asymptotic behavior of quadratic functions. Jung [9] has proved an asymptotic property of quadratic functions (see also [25]).

Corollary 4.4 Let $f: X \rightarrow Y$. Then the following statements are equivalent:

(i) $\lim \sup _{\|x\|+\|y\| \rightarrow \infty}\|f(2 x-y)+f(x+2 y)-5 f(x)-5 f(y)\|_{\beta}=0$;

(ii) $\lim \sup _{\max \{\|x\|,\|y\|\} \rightarrow \infty}\|f(2 x-y)+f(x+2 y)-5 f(x)-5 f(y)\|_{\beta}=0$;

(iii) $f(2 x-y)+f(x+2 y)=5 f(x)+5 f(y), x, y \in X$;

(iv) $f$ is quadratic. 
Proof Since $\max \{\|x\|,\|y\|\} \leq\|x\|+\|y\| \leq 2 \max \{\|x\|,\|y\|\}$, conditions (i) and (ii) are equivalent. The equivalence of (iii) and (iv) and (iv) $\Rightarrow$ (i) follows from Theorem 2.1. To prove (i) $\Rightarrow$ (iv), by the assumption there exists an increasing sequence of positive numbers $\left\{d_{n}\right\}_{n=1}^{\infty}$ such that

$$
\|f(2 x-y)+f(x+2 y)-5 f(x)-5 f(y)\|_{\beta} \leq \frac{1}{n}, \quad\|x\|+\|y\| \geq d_{n} .
$$

By Theorem 4.2 there exists a unique quadratic function $Q_{n}: X \rightarrow Y$ such that

$$
\left\|f(x)-Q_{n}(x)\right\|_{\beta} \leq \frac{c}{n}, \quad x \in X, n \in \mathbb{N}
$$

where $c:=\frac{9^{\beta}+5^{\beta}+1}{5^{\beta}\left(9^{\beta}-1\right)}$. Since $Q_{n}$ is a unique quadratic function satisfying (4.6) and

$$
\left\|f(x)-Q_{n+1}(x)\right\|_{\beta} \leq \frac{c}{n+1} \leq \frac{c}{n}, \quad x \in X, n \in \mathbb{N},
$$

we infer that $Q_{n+1}=Q_{n}$ for all $n \in \mathbb{N}$. Therefore (4.6) implies that

$$
\left\|f(x)-Q_{1}(x)\right\|_{\beta}=\left\|f(x)-Q_{n}(x)\right\|_{\beta} \leq \frac{c}{n}, \quad x \in X, n \in \mathbb{N} .
$$

Taking the limit as $n \rightarrow \infty$, we obtain $f(x)=Q_{1}(x)$ for all $x \in X$. Hence $f$ is quadratic.

Corollary 4.5 Let $f: X \rightarrow Y$, and let $z$ be a fixed point of $Y$. Then the following statements are equivalent:

(i) $\limsup _{\|x\|+\|y\| \rightarrow \infty}\|f(2 x-y)+f(x+2 y)-5 f(x)-5 f(y)-z\|_{\beta}=0$;

(ii) $\limsup _{\max \{\|x\|,\|y\|\} \rightarrow \infty}\|f(2 x-y)+f(x+2 y)-5 f(x)-5 f(y)-z\|_{\beta}=0$;

(iii) $f(2 x-y)+f(x+2 y)=5 f(x)+5 f(y)+z, x, y \in X$;

(iv) $f+\frac{1}{8} z$ is quadratic, that is,

$$
f(x+y)+f(x-y)=2 f(x)+2 f(y)+\frac{1}{4} z, \quad x, y \in X .
$$

Proof Define $g: X \rightarrow Y$ by $g(x):=f(x)+\frac{1}{8} z$. Clearly,

$$
f(2 x-y)+f(x+2 y)-5 f(x)-5 f(y)-z=g(2 x-y)+g(x+2 y)-5 g(x)-5 g(y)
$$

for all $x, y \in X$. Then (i)-(iv) are equivalent by Corollary 4.4 .

Corollary 4.6 Let $f: X \rightarrow Y$, and let $z$ be a fixed point of $Y$. Then the following statements are equivalent:

(i) $f(2 x-y)+f(x+2 y)-5 f(x)-5 f(y)=z,\|x\|+\|y\| \geq d$ for some $d>0$;

(ii) $f(2 x-y)+f(x+2 y)=5 f(x)+5 f(y)+z, x, y \in X$;

(iii) $f+\frac{1}{8} z$ is quadratic.

Corollary 4.7 Let $d>0$ and $\delta \geq 0$. Assume that a function $f: X \rightarrow Y$ satisfies the inequality

$$
\|f(2 x-y)+f(x+2 y)-5 f(x)-5 f(y)\|_{\beta} \leq \delta
$$


for all $(x, y) \in X^{2} \backslash E$, where $E:=\left\{(x, y) \in X^{2}:\|x\|<d,\|y\|<d\right\}$. Then there exists a unique quadratic function $Q: X \rightarrow Y$ satisfying (4.2).

Proof It is clear that $\left\{(x, y) \in X^{2}:\|x\|+\|y\| \geq 2 d\right\} \subseteq X^{2} \backslash E$. Hence the result follows from Theorem 4.2.

Using the same argument as in Theorem 4.2, we obtain the following result.

Theorem 4.8 Let $d>0$ and $\delta \geq 0$. Assume that a function $f: X \rightarrow Y$ satisfies the inequality

$$
\|f(2 x-y)+f(x+2 y)-5 f(x)-5 f(y)\|_{\beta} \leq \delta, \quad\|x\| \geq d(\text { respectively, }\|y\| \geq d) .
$$

Then there exists a unique quadratic function $Q: X \rightarrow Y$ satisfying (4.2) for all $x \in X$.

Corollary 4.9 Let $f: X \rightarrow Y$, and let $z$ be a fixed point of $Y$. Then the following statements are equivalent:

(i) $\limsup _{\|x\| \rightarrow \infty}\|f(2 x-y)+f(x+2 y)-5 f(x)-5 f(y)-z\|_{\beta}=0$;

(ii) $\lim \sup _{\|y\| \rightarrow \infty}\|f(2 x-y)+f(x+2 y)-5 f(x)-5 f(y)-z\|_{\beta}=0$;

(iii) $f(2 x-y)+f(x+2 y)=5 f(x)+5 f(y)+z$ for all $x, y \in X$;

(iv) $f+\frac{1}{8} z$ is quadratic.

Proof Without loss of generality, we may assume that $z=0$. It suffices to prove (i) $\Rightarrow$ (iv) and (ii) $\Rightarrow$ (iv). We only prove (i) $\Rightarrow$ (iv), and (ii) $\Rightarrow$ (iv) is similarly achieved. To prove (i) $\Rightarrow$ (iv), by the assumption there exists an increasing sequence of positive numbers $\left\{d_{n}\right\}_{n=1}^{\infty}$ such that

$$
\|f(2 x-y)+f(x+2 y)-5 f(x)-5 f(y)\|_{\beta} \leq \frac{1}{n}, \quad\|x\| \geq d_{n} .
$$

By Theorem 4.8 there exists a unique quadratic function $Q_{n}: X \rightarrow Y$ such that

$$
\left\|f(x)-Q_{n}(x)\right\| \leq \frac{c}{n}, \quad x \in X, n \in \mathbb{N},
$$

where $c:=\frac{9^{\beta}+5^{\beta}+1}{5^{\beta}\left(9^{\beta}-1\right)}$. The rest of the proof is quite similar to that of Corollary 4.4, and we leave it.

Corollary 4.10 Let $f: X \rightarrow Y$, and let $z$ be a fixed point of $Y$. Then the following statements are equivalent:

(i) $f(2 x-y)+f(x+2 y)-5 f(x)-5 f(y)=z,\|x\| \geq d$ for some $d>0$;

(ii) $f(2 x-y)+f(x+2 y)-5 f(x)-5 f(y)=z,\|y\| \geq c$ for some $c>0$;

(iii) $f(2 x-y)+f(x+2 y)-5 f(x)-5 f(y)=z$ for all $x, y \in X$;

(iv) $f+\frac{1}{8} z$ is quadratic. 
Availability of data and materials

Not applicable.

\section{Competing interests}

The authors declare that they have no competing interests.

\section{Authors' contributions}

The authors equally conceived of the study, participated in its design and coordination, drafted the manuscript, participated in the sequence alignment, and read and approved the final manuscript.

\section{Author details}

'Humanitas College, Kyung Hee University, Yongin 17104, Republic of Korea. ${ }^{2}$ Department of Mathematics, Faculty of Sciences, University of Mohaghegh Ardabili, Ardabil, Iran.

\section{Publisher's Note}

Springer Nature remains neutral with regard to jurisdictional claims in published maps and institutional affiliations.

Received: 7 May 2020 Accepted: 16 February 2021 Published online: 27 February 2021

\section{References}

1. Aczél, A., Dhombres, H.: Functional Equations in Several Variables. Cambridge University Press, Cambridge (1989)

2. Hyers, D.H., Isac, G., Rassias, Th.M.: Stability of Functional Equations in Several Variables. Birkhäuser, Basel (1998)

3. Kannappan, PI.: Quadratic functional equation and inner product spaces. Results Math. 27, 368-372 (1995)

4. Jordan, P., von Neumann, J.: On inner products in linear metric spaces. Ann. Math. 36, 719-723 (1935)

5. Skof, F.: Proprieta locali e approssimazione di operatori. Rend. Semin. Mat. Fis. Milano 53, 113-129 (1983)

6. Czerwik, S.: On the stability of the quadratic mapping in normed spaces. Abh. Math. Semin. Univ. Hamb. 62, 59-64 (1992)

7. Cholewa, P.W.: Remarks on the stability of functional equations. Aequ. Math. 27, 76-86 (1984)

8. Jun, K.-W., Lee, Y.-H.: On the Hyers-Ulam-Rassias stability of a Pexiderized quadratic inequality. Math. Inequal. Appl. 4, 93-118 (2001)

9. Jung, S.-M.: On the Hyers-Ulam stability of the functional equations that have the quadratic property. J. Math. Anal. Appl. 222, 126-137 (1998)

10. Lee, Y.-H., Jung, S.-M., Rassias, M.Th.: Uniqueness theorems on functional inequalities concerning cubic-quadratic-additive equation. J. Math. Inequal. 12, 43-61 (2018)

11. Lee, Y.-H., Jung, S.-M., Rassias, M.Th.: On an $n$-dimensional mixed type additive and quadratic functional equation. Appl. Math. Comput. 228, 13-16 (2014)

12. Moghimi, M.B., Najati, A., Park, C.: A functional inequality in restricted domains of Banach modules. Adv. Differ. Equ. 2009, Art. ID 973709, 14 pp. (2009)

13. Najati, A.: Hyers-Ulam stability of an $n$-Apollonius type quadratic mapping. Bull. Belg. Math. Soc. Simon Stevin 14, 755-774 (2007)

14. Najati, A., Park, C.: Hyers-Ulam-Rassias stability of homomorphisms in quasi-Banach algebras associated to the Pexiderized Cauchy functional equation. J. Math. Anal. Appl. 335, 763-778 (2007)

15. Najati, A., Park, C.: The Pexiderized Apollonius-Jensen type additive mapping and isomorphisms between $C^{*}$-algebras. J. Differ. Equ. Appl. 14, 459-479 (2008)

16. Noori, B., Moghimi, M.B., Khosravi, B., Park, C.: Stability of some functional equations on bounded domains. J. Math. Inequal. 14, 455-471 (2020)

17. Rassias, J.M.: On the Ulam stability of mixed type mappings on restricted domains. J. Math. Anal. Appl. 276, 747-762 (2002)

18. Czerwik, S.: Functional Equations and Inequalities in Several Variables. World Scientific, Singapore (2002)

19. Jung, S.-M.: Hyers-Ulam-Rassias Stability of Functional Equations in Nonlinear Analysis. Springer, New York (2011)

20. Kannappan, PI.: Functional Equations and Inequalities with Applications. Springer, Berlin (2009)

21. Sahoo, P.K., Kannappan, PI.: Introduction to Functional Equations. Taylor \& Francis, London (2011)

22. Šemrl, P.: Additive functions and two characterizations of inner-product spaces. Glas. Mat. Ser. III 25(45), 309-317 (1990)

23. Diaz, J., Margolis, B.: A fixed point theorem of the alternative for contractions on a generalized complete metric space. Bull. Am. Math. Soc. 74, 305-309 (1968)

24. Cădariu, L., Radu, V.: Fixed points and the stability of quadratic functional equations. An. Univ. Vest. Timişs., Ser. Mat.-Inform. 41, 25-48 (2003)

25. Najati, A., Jung, S.-M.: Approximately quadratic mappings on restricted domains. J. Inequal. Appl. 2010, Article ID $503458(2010)$ 